\title{
The Many C's of Primary Care
}

Despite a rather pendular history, primary care remains the axle of any health care system, from which all other spokes radiate. As originally defined by the Institute of Medicine, primary care physicians (PCPs) provide continuous, comprehensive, coordinated care in addition to serving as the first point of contact for most health care needs. ${ }^{1}$ Table 1 delineates additional C's which, though not unique to primary care, adhere particularly strongly to the generalist. Paradoxically, the many C's both encumber and empower the PCP. The final entry in Table 1 -competing demands-is sort of a sum total of the preceding C's, connoting the complexity of primary care practice. Omitted from Table 1 is controllable life style-a $\mathrm{C}$ that may be relatively deficient in primary care, thereby deflecting a number of medical students to other specialty career choices. ${ }^{2}$

The articles in this issue of Journal of General Internal Medicine collectively address a number of the C's. Physical symptoms account for half of all outpatient visits, are medically unexplained at least one third of the time, and are frequently comorbid with depression, anxiety, and other psychosocial factors. ${ }^{3}$ In the two thirds of depressed patients who presented exclusively with physical symptoms, Keeley et al. found deficits in communication and compliance: somatizers were asked less often about depression and, if started on antidepressants, were less likely to complete an adequate trial. ${ }^{4}$ The fact that depression presents in primary care predominantly in the guise of physical symptoms is a global phenomenon. ${ }^{5}$ The depression intervention was less effective in patients presenting with exclusively physical symptoms. The authors conclude that such patients may require increased pharmacotherapy doses, duration, and/or different drugs or specific psychotherapy. However, physical presenters were also less likely than patients with psychological symptoms to consider antidepressants an acceptable treatment (40\% vs $72 \%$ ). Such skepticism could have contributed to a poorer antidepressant response. Some physical presenters may also be unconvinced that depression fully explains their symptoms. In this case, reattribution may be part of the treatment. ${ }^{6}$ Finally, adjunctive treatment for physical symptoms (e.g., analgesics for pain) may be required.

The importance of continuity of care was demonstrated in two studies. Doescher et al. found that site continuity enhanced rates of influenza vaccination and mammography, and that provider continuity provided additional enhancement. ${ }^{7}$ The authors go on to say: "Provider continuity is associated with greater trust, and trust may be linked to greater adherence to physician recommendations. In addition, continuity relationships may also allow providers to sort more effectively through the multiple competing demands posed by patients and adequately address prevention."

Ten C's of Primary Care

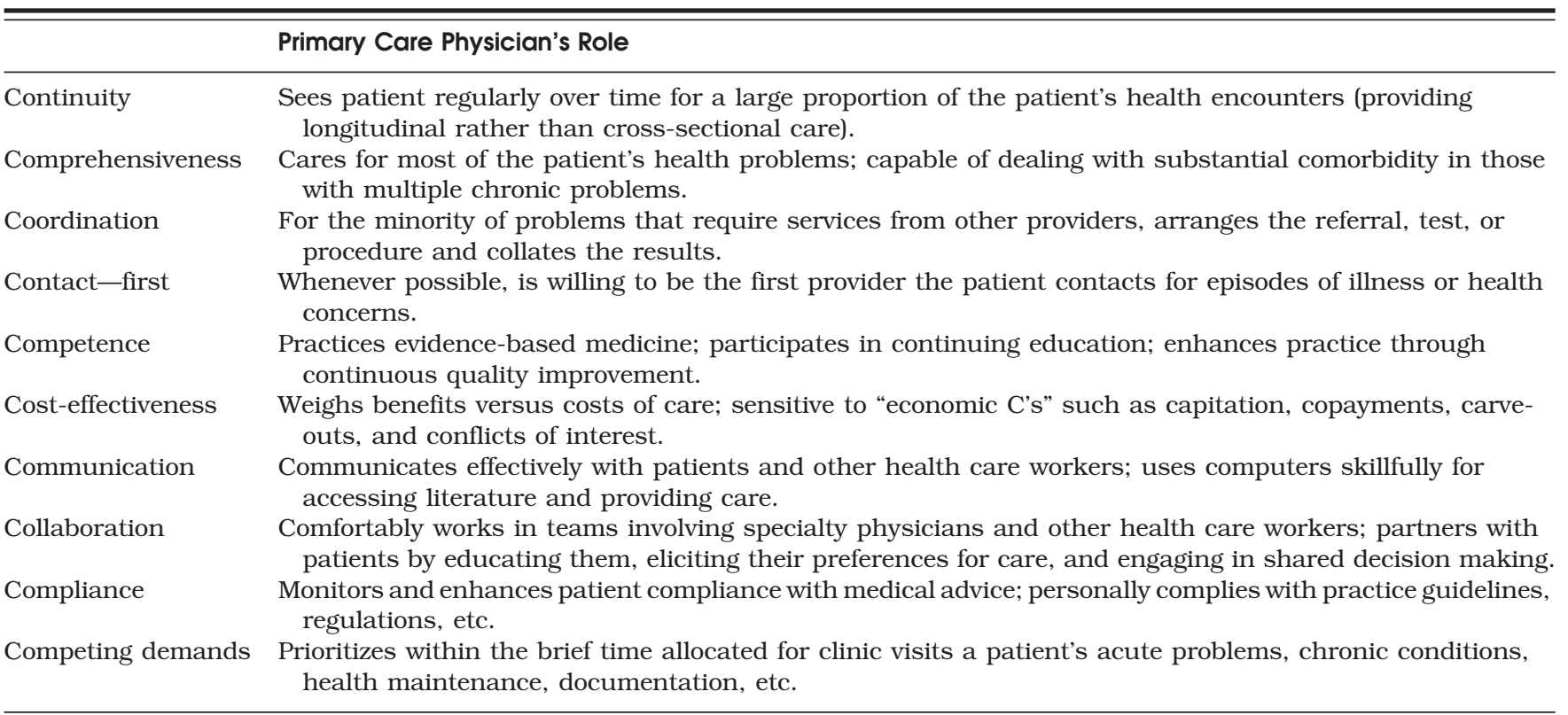


In a novel (yet not entirely surprising) twist to our standard concept of PCP-based continuity of care, van Walraven et al. found that outcomes were better if patients were followed up after hospital discharge by the physician who had provided their inpatient care rather than by their usual outpatient physician. ${ }^{8}$ Admittedly, the benefits were modest and would need to be confirmed in a trial randomizing discharged patients to follow-up by the hospital physician versus follow-up with their PCP who is provided complete information about the hospitalization. Indeed, improved transfer of care from inpatient to outpatient physicians has been identified as a priority area for research.

Competing demands can be onerous. ${ }^{9}$ For example, a single disease such as uncomplicated diabetes may be governed by practice guidelines with up to 11 clinical triggers for interventions. Hofer et al. discovered that when faced with "too much to do," physicians undervalued some interventions for type 2 diabetes recently established as beneficial while overvaluing other interventions which because of ease of measurement are favored as performance measures. ${ }^{10}$ Endocrinologists did slightly better than PCPs. The authors note that "for a routine primary care visit of 10 minutes, the average patient has 15.4 risk factors and 24.5 recommendations based on the United States Preventive Services Task Force guidelines. Because many people also have chronic diseases, the competing demands problem is actually much larger, even before considering acute injury and illness."

Ellis et al. found that suboptimal statin adherence and discontinuation was similar in both primary and secondary prevention patient populations and was adversely influenced by the level of patient copayment. ${ }^{11}$ They concluded that "incremental efforts, including those that decrease out-of-pocket pharmaceutical expenditures, should focus on improving adherence in high-risk populations most likely to benefit from statin use." The subtitle of their article-"Should We Target Patients with the Most to Gain?"-is a question germane to many of our competing choices in health care.

Swarztrauber and Vickrey evaluated specialty preferences regarding care of elderly patients with neurological conditions. ${ }^{12}$ For all three scenarios, neurologists preferred a greater extent of specialty involvement compared to PCPs. The authors concluded that their findings raise questions about collaboration and coordination of care. Interestingly, they only chose scenarios of patients with distinct diagnoses and evidence-based treatments. What the specialist often promptly volleys back to the PCP is the patient with chronic symptoms. Some of these patients have undiagnosed psychological disorders, as noted by Keeley et al. Others have functional somatic syndromes (e.g., irritable bowel syndrome, fibromyalgia, chronic fatigue) or simply "medically unexplained" symptoms. All require effective doctor-patient communication, judicious use of tests and treatments, and collaboration between PCPs and specialists.

Of all the attributes of primary care valued by patients, continuity ranks among the highest. ${ }^{13}$ However, the various C's augment one another. Coordinated, collaborative care with effective communication that triangulates patients, PCPs, and specialists is preferable to a "PCP versus specialist" debate. For example, postmyocardial infarction outcomes are better for patients receiving care from both PCPs and subspecialists than for patients seen by specialists or PCPs alone. ${ }^{14}$ The future of general internal medicine is under scrutiny. ${ }^{15}$ However, any reshaping of the discipline must consider the many C's upon which primary care is founded.-Kurt KRoenKe, MD, Department of Medicine, Regenstrief Institute and Indiana University, Indianapolis, Ind.

\section{References}

1. Sox HC. The future of primary care. Ann Intern Med. 2003;138:230-1.

2. Dorsey ER, Jarjoura D, Rutecki GW. Influence of controllable lifestyle on recent trends in specialty choice by US medical students. JAMA. 2003;290:1173-8.

3. Kroenke K. The interface between physical and psychological symptoms. Prim Care Companion J Clin Psychiatry. 2003;5(suppl 7):11-8.

4. Keeley RD, Smith JL, Nutting PA, Dickinson LM, Dickinson WP, Rost KM. Does a depression intervention result in improved outcomes for patients presenting with physical symptoms? J Gen Intern Med. 2004;19:615-23.

5. Simon GE, Von Korff M, Picinelli M, Fullerton C, Ormel J. An international study of the relation between somatic symptoms and depression. N Engl J Med. 1999;341:1329-35.

6. Morriss RK, Gask L. Treatment of patients with somatized mental disorder: effects of reattribution training on outcomes under the direct control of the family doctor. Psychosomatics. 2002;43:394-9.

7. Doescher MP, Saver BG, Fiscella K, Franks P. Preventive care: does continuity count? J Gen Intern Med. 2004;19:632-7.

8. van Walraven C, Mamdani M, Fang J, Austin PC. Continuity of care and patient outcomes after hospital discharge. J Gen Intern Med. 2004;19:624-31.

9. Klinkman MS. Competing demands in psychosocial care: a model for the identification and treatment of depressive disorders in primary care. Gen Hosp Psychiatry. 1997;19:98-111.

10. Hofer TP, Zemencuk JK, Hayward RA. When there is too much to do: how practicing physicians prioritize among recommended interventions. J Gen Intern Med 2004;19:646-53.

11. Ellis JJ, Erickson SR, Stevenson JG, Bernstein SJ, Stiles RA, Fendrick AM. Suboptimal statin adherence and discontinuation in primary and secondary prevention populations: should we target patients with the most to gain? J Gen Intern Med 2004;19:638-45.

12. Swarztrauber K, Vickrey BG. Do neurologists and primary care physicians agree on the extent of specialty involvement of patients referred to neurologists? J Gen Intern Med. 2004;19:654-61.

13. Moore G, Showstack J. Primary care medicine in crisis: toward reconstruction and renewal. Ann Intern Med. 2003;138:244-7.

14. Ayanian JZ, Landrum MB, Guadagnoli E, Gaccione P. Specialty of ambulatory care physicians and mortality among elderly patients after myocardial infarction. N Engl J Med. 2002;547:1678-86.

15. Larson EB, Fihn SD, Kirk LM, et al. The future of general internal medicine: report and recommendations from the Society of General Internal Medicine (SGIM) Task Force on the Domain of General Internal Medicine. J Gen Intern Med. 2004;19:69-77. 